\title{
Coherent interferometric dual frequency laser radar for precise range/Doppler measurement
}

\author{
Daniel Onori, Filippo Scotti, Mirco Scaffardi, Antonella Bogoni, and Francesco Laghezza
}

\begin{abstract}
A novel coherent interferometric dual frequency laser radar that merges the concept of laser and radio detection and ranging (ladar and radar respectively), for both range and velocity measurement, is presented and experimentally demonstrated. The innovative architecture combines the broadband tunability of dual-wavelength optical sources, enabling a dynamic trade-off between precision and robustness in Doppler estimation, with the high stability of low frequency RF sources for the interferometric measure of the target range with extreme accuracy. The possibility to easily reconfigure the employed frequencies, allows to change the Doppler resolution, as well as the range ambiguity and precision, to dynamically adapt the system to reliably operate in different environments. Moreover, the coherent detection allows to enhance the signal to noise ratio reaching excellent performances also with low level of received power. The laboratory characterization provides an estimation of the system performances, in terms of resolution and sensitivity, as well as the indoor demonstration with targets of opportunity proves the effectiveness of the proposed architecture to operate in real scenarios.
\end{abstract}

Index Terms-laser radar, coherent detection, range and Doppler measurements.

\section{INTRODUCTION}

$\mathrm{O}$ VER recent years, thanks to the innovation in optical and electro-optical components, light (or laser) detection and ranging (LIDAR or LADAR) systems are having great success, proving themselves as a considerable alternative to passive radiometers or microwave sensors [1]. LADAR sensor emits energy in the optical frequency range (many order of magnitude greater than radars) retrieving information about the target of interest by collecting the backscattered light. Although the principle of operation is the same of radar systems, there are many differences between light and microwave sensing. For example, radars are widely used in all day/weather conditions being robust to atmospheric turbulences, also demonstrating penetration capabilities. Nevertheless they have poor cross range resolution, lack in velocity estimation of slow targets, and are vulnerable to radio interference sources also demonstrating high probability of

Manuscript received on January 15, 2016.

This work has been carried on within EU projects RAPIDO, ROAM, PETRA, and FiWin5G.

D. Onori is with Scuola Superiore Sant'Anna - TECIP Insitute, Pisa, Italy (daniel.onori@sssup.it).

F. Scotti, M. Scaffardi, A. Bogoni, and F. Laghezza are with CNIT National Laboratory of Photonic Networks, Pisa, Italy. intercept [2]. Besides, LADAR systems allow higher directionality thanks to the very narrow beam divergence capable to solve small targets, and are a limited source of interferences with low probability of intercept. Nevertheless they present high sensibility to the atmospheric condition, and a limited range because of particle absorption [3]. LADAR applications include target detection and ranging, gas and particle size [4] detection and recognition (via differential absorption and scattering), vibrometry [5], imaging [6], synthetic aperture imaging [7] and more over. Depending on the application, LADARs can exploit incoherent or coherent detection. Optically incoherent schemes, which resort to intensity modulation and direct detection (IMDD), are only used for range extraction, while optically coherent architectures are used to obtain both range and Doppler measurements, exploiting the phase information of the optical wave. [8]. Moreover, IMDD systems that resort to amplification stages at the receiver are limited by the amplified spontaneous emission (ASE) noise, thus one of the main advantages of optically coherent detection can be identified in an amplification gain which allows to increase the signal to noise ratio (SNR) and the sensitivity of the system.

Three different incoherent techniques are often used to extract range information of the targets: pulses time of flight distance measurement, phase shift range finder and phaselfrequency modulated pulses [9]. The first one is based on short pulse emission and the relative measurement of the round trip time between transmission and echo reception. Range resolution and ambiguity directly depend on pulse width and repetition interval while precision also depends on receiver noise contributions. The phase shifts technique is based on optical power modulation via a constant radio frequency tone at the transmitter side, and phase detection at the receiver. The selection of the radio frequency carrier determines the maximum range ambiguity of the system, while signal to noise ratio (SNR) influences the phase estimation and consequently the range precision. Going forward, in phaselfrequency modulation laser radar system, the basic principle of pulse compression technique is exploited to increase the range resolution. For example linear or non-linear frequency modulated optical carriers are used to determine target range trough a beat frequency estimation between a reference and the backscattered optical signals. The time bandwidth product of the employed waveform guarantees good range resolution performances. Other examples of different modulation techniques, such as stepped frequency pulses, complementary 
code generation, and sparse frequency chirp are detailed in [10],[11],[12].

On the other hand, a direct measurement of the velocity can be reached by using coherent LADAR systems, which measure the Doppler shift experienced by the optical beam. They are widely used for atmospheric sensing [13], wake vortex risk mitigation [14],[15] aerosol, clouds, gases tracking [16], and more besides. Basically, in a coherent laser radar architecture, the back reflected light from a target is compared with a nominal reference wavelength, thus determining the occurred Doppler shift [17][18]. Nevertheless the speckle noise, induced by target's roughness and atmospheric turbulence [19], causes spectral broadening of the Doppler shifted line, thus limiting the spectral resolution of the sensing system [20]. In order to reach high robustness against the speckle noise, coherent dual-frequency lidar (DFL) architectures are exploited [21]. In these systems, the measurement of the Doppler shift is obtained as a difference among the Doppler shift of two locked continuous waves. In all the coherent techniques, the maximum observable range is relative to the coherence length of the employed laser source, thus ultra stable optical sources, e.g. mode-locked lasers (MLL) [22], are used to extend the monitored range. The authors in [23] investigated an architecture in which different couples of modes from a MLL are used to emulate different microwave frequencies for Doppler detection. Nevertheless, such implementation is not able to provide information about the target's distance.

In this paper we propose and implement, for the first time to out knowledge, a coherent interferometric dual frequency laser radar (IDFL) for simultaneous velocity and range measurements. The architecture resort to the principle of the DFLs to perform Doppler processing and to the principle of differential interferometric phase estimation for the extraction of the range information. The details of the principle of operation are described in section II. The laser radar demonstrator has been tested with cooperative targets and the obtained results, for both range and speed measurements, are presented and discussed (section III), demonstrating the effectiveness of the proposed approach.

\section{PRINCIPLE OF OPERATION}

The proposed architecture exploits two phase locked optical carriers, modulated by a low frequency RF tone, to gather information about both distance and speed of the illuminated target. The scheme of the operation principle is depicted in Fig.1. The two optical carriers are generated by a tunable dual frequency laser source (T-DFL), which could be implemented resorting, e.g., to a coherent frequency comb [24], a MLL [23], or directly to a dual frequency laser [25]. The two modes present electrical fields $E_{1}(t)$ and $E_{2}(t)$ centered at the optical frequencies $v_{1}$ and $v_{2}$, respectively, separated by $\Delta f$ (Fig.1-A). The resulting electrical field $E(t)$ can be expressed as:

$$
E(t)=E_{1}(t)+E_{2}(t)=A_{1} \exp \left(i \cdot 2 \pi v_{1} t\right)+A_{2} \exp \left(i \cdot 2 \pi v_{2} t\right)+c . c .
$$

where $A_{1}$ and $A_{2}$ are the amplitudes of the two carriers. For simplicity their phases are supposed equal and null. This optical signal is then split and sent to the transmission and reference path respectively. The reference replica is frequency shifted by $f_{I F}$, as represented in Fig.1-B, in order to be able of evaluating the sign of the target velocity, and can be written as

$$
E_{R E F}(t)=\frac{A_{1}}{\sqrt{2}} \exp \left[i \cdot 2 \pi\left(v_{1}+f_{I F}\right) t\right]+\frac{A_{2}}{\sqrt{2}} \exp \left[i \cdot 2 \pi\left(v_{2}+f_{I F}\right) t\right]+c . c .
$$

The other replica is modulated in a Mach-Zehnder modulator (MZM), biased in carrier suppression mode, by a RF tone with frequency $f_{\mathrm{M}}$ and, for simplicity of notation, phase $\varphi_{\mathrm{M}}=0$. Four optical tones are thus created as depicted in Fig.2-C, so that their electrical field can be represented as:

$$
\begin{aligned}
& E_{M}(t)=B_{1} \exp \left[i \cdot 2 \pi\left(v_{1}-f_{M}\right) t\right]+B_{1} \exp \left[i \cdot 2 \pi\left(v_{1}+f_{M}\right) t\right]+ \\
& +B_{2} \exp \left[i \cdot 2 \pi\left(v_{2}-f_{M}\right) t\right]+B_{2} \exp \left[i \cdot 2 \pi\left(v_{2}+f_{M}\right) t\right]+c . c .
\end{aligned}
$$

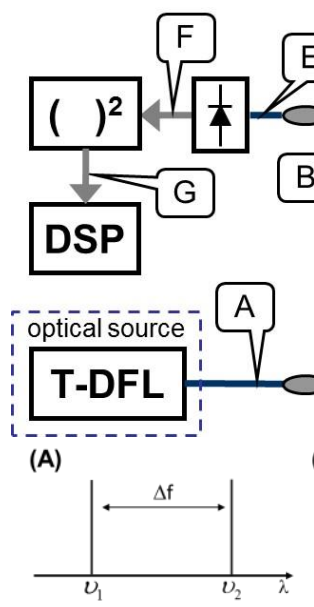

(D)

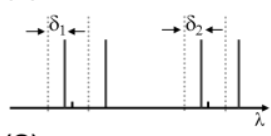

(E)

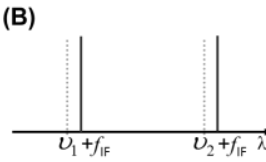

(B)

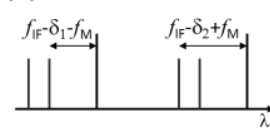

(F)

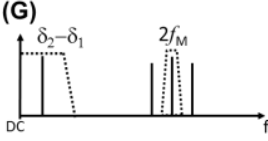

Fig. 1. Top: scheme of principle of the proposed architecture-DFL tunable dual frequency laser, MZM Mach Zehnder modulator, DSP digital signal processing. Bottom: Optical and electrical spectra at different points of the scheme of principle.

with $B_{1}$ and $B_{2}$ amplitude terms. These two couples of modes, separated by a frequency $2 f_{M}$, will be exploited for the interferometric estimation of the target range. Furthermore, since their central frequencies $v_{1}$ and $v_{2}$ are in turn separated by $\Delta f$, the experienced differential Doppler can also be estimated [23]. This signal is transmitted through a telescope to the target which reflects it back. The backscattered signal, after experiencing a time delay and a Doppler shift (Fig.2-D), can be written as:

$$
\begin{aligned}
E_{B S}(t) & =C_{1} \exp \left[i \cdot 2 \pi\left(v_{1}-f_{M}+\delta_{1 A}\right) t-i \cdot 2 \pi\left(v_{1}-f_{M}\right) \tau\right]+ \\
& +C_{1} \exp \left[i \cdot 2 \pi\left(v_{1}+f_{M}+\delta_{1 B}\right) t-i \cdot 2 \pi\left(v_{1}+f_{M}\right) \tau\right]+ \\
& +C_{2} \exp \left[i \cdot 2 \pi\left(v_{2}-f_{M}+\delta_{2 A}\right) t-i \cdot 2 \pi\left(v_{2}-f_{M}\right) \tau\right]+ \\
& +C_{2} \exp \left[i \cdot 2 \pi\left(v_{2}+f_{M}+\delta_{2 B}\right) t-i \cdot 2 \pi\left(v_{2}+f_{M}\right) \tau\right]+c . c .
\end{aligned}
$$

where $\tau$ is the relative delay induced by the target position, $C_{I}$ and $C_{2}$ represent the fields amplitudes, and $\delta_{1 \mathrm{~A}}, \delta_{1 \mathrm{~B}}, \delta_{2 \mathrm{~A}}$, and $\delta_{2 \mathrm{~B}}$ 
represent the Doppler shifts, functions of the target velocity as well as the modes frequencies. In detail

$$
\begin{aligned}
& \delta_{1 A}=2 \varepsilon\left(v_{1}-f_{M}\right) / c \\
& \delta_{1 B}=2 \varepsilon\left(v_{1}+f_{M}\right) / c \\
& \delta_{2 A}=2 \varepsilon\left(v_{2}-f_{M}\right) / c \\
& \delta_{2 B}=2 \varepsilon\left(v_{2}+f_{M}\right) / c
\end{aligned}
$$

where $\varepsilon$ represents the target speed and $c$ is the speed of light. Since $f_{\mathrm{M}}<<\Delta f$ we can consider $\delta_{1 \mathrm{~A}} \approx \delta_{1 \mathrm{~B}}=\delta_{1}$ and $\delta_{2 \mathrm{~A}} \approx \delta_{2 \mathrm{~B}}=\delta_{2}$. An optical coupler combines echo signal $E_{\mathrm{BS}}(t)$ together with the reference beam $E_{R E F}(t)$, as shown in Fig.2-E. The re-coupled signal is sent to a photodiode (PD) where the tones are heterodyned together. Among all the generated terms, only five tones fold down to the analog to digital converter (ADC) bandwidth (Fig.1-F), and can be digitized. Four of these tones are generated by the coherent beating of $E_{\mathrm{BS}}(t)$ with $E_{R E F}(t)$ and their amplitudes are proportional to the amplitude of $E_{R E F}(t)$, thus performing a coherent amplification of the backscattered signal. The fifth term instead is the direct detection of the modes separated by $2 f_{M}$, which is very weak with respect to the coherent ones and can be neglected or filtered out. The output signal of the photodiode $I_{\mathrm{PD}}(\mathrm{t})$ can be written as

$$
\begin{aligned}
& I_{P D}(t)=D_{1} \exp \left[i \cdot 2 \pi\left(f_{I F}+f_{M}-\delta_{1}\right) t-i \cdot 2 \pi\left(v_{1}-f_{M}\right) \tau\right]+ \\
& +D_{1} \exp \left[i \cdot 2 \pi\left(f_{I F}-f_{M}-\delta_{1}\right) t-i \cdot 2 \pi\left(v_{1}+f_{M}\right) \tau\right]+ \\
& +D_{2} \exp \left[i \cdot 2 \pi\left(f_{I F}+f_{M}-\delta_{2}\right) t-i \cdot 2 \pi\left(v_{2}-f_{M}\right) \tau\right]+ \\
& +D_{2} \exp \left[i \cdot 2 \pi\left(f_{I F}-f_{M}-\delta_{2}\right) t-i \cdot 2 \pi\left(v_{2}+f_{M}\right) \tau\right]+c . c .
\end{aligned}
$$

with $D_{1}$ and $D_{2}$ amplitude terms. These four terms suffer the previously mentioned spectral broadening due to speckle and ambient noise, and their frequencies can not directly provide a precise measure of the velocity of the target. Moreover to prevent aliasing and overlapping of the different frequency components, the condition $\left(f_{\mathrm{IF}}+f_{\mathrm{M}}\right)>\delta_{\mathrm{MAX}}$ has to be satisfied, where $\delta_{\text {MAX }}$ is the maximum expected Doppler shift.

A square law operation on to the electrical signal $I_{\mathrm{PD}}(t)$ allows us to obtain the desired information of the target range and speed. Among all the generated terms, only the ones at lower frequencies are of interest, as depicted in Fig.1-G. In particular the term

$$
P_{r}(t)=\left(D_{1}^{2}+D_{2}^{2}\right) \cdot \exp \left[i \cdot 2 \pi\left(2 f_{M}\right) t-i \cdot 2 \pi\left(2 f_{M}\right) \tau\right]
$$

contains the time delay information from which the target range can be extracted, and the term

$$
P_{v}(t)=2\left(D_{1} \cdot D_{2}\right) \cdot \exp \left[i \cdot 2 \pi\left|\delta_{2}-\delta_{1}\right| t+i \cdot 2 \pi\left(v_{2}-v_{1}\right) \tau\right]
$$

contains the information related to the differential Doppler $\mid \delta_{2}$ $-\delta_{1} \mid$ used for the measurement of the target velocity, while the phase term is ignored.

Assuming $\left|\delta_{2}-\delta_{1}\right|<f_{\mathrm{M}}$, the terms $P_{r}(t)$ and $P_{v}(t)$ can be extracted by means of a narrow digital band pass filter centered at $2 f_{M}$, and a low pass filter spanning from DC to $f_{M}$, respectively, as shown in Fig.2-G.

The range processing resorts to the phase term of (7) induced by the delay $\tau$, given by

$$
\varphi_{r}=2 \pi\left(2 f_{M}\right) \tau
$$

The phase difference $\Delta \varphi=\varphi_{r}-\varphi_{M}$ provides the information about the target range $R$, and since the phase $\varphi_{M}$ of the RF tone has been set equal to zero the target distance is:

$$
R=\frac{c}{2} \cdot \tau=\frac{c}{2} \cdot\left[\frac{\varphi_{r}}{4 \pi \cdot f_{M}}\right]
$$

with an unambiguous range $R_{N A}$ function of $f_{\mathrm{M}}$, expressed as

$$
R_{N A}=\frac{c}{2\left(2 f_{M}\right)}
$$

Concerning the target velocity measure, referring to (8), the estimation of the frequency $\left|\delta_{2}-\delta_{1}\right|$ of the signal $P_{\mathrm{v}}(t)$ provides information about the target speed $\varepsilon$ without additional noise induced by target roughness and atmospheric turbulence [21]. Nevertheless the square law operation does not preserve the information of the movement direction (the sing of $\left(\delta_{2}-\delta_{1}\right)$ ), thus the resulting velocity information is

$$
|\varepsilon|=\frac{\left|\delta_{2}-\delta_{1}\right| c}{2 \Delta f}
$$

The sign of the velocity vector $\varepsilon$ can be recovered from (6) evaluating the direction of the frequency shifts experienced by the four tones due to the Doppler effect (see Fig.1-F).

\section{EXPERIMENTAL SETUP AND RESULTS}

To validate the concepts detailed in the previous section, the proposed laser radar has been implemented. The developed experimental setup is sketched in Fig.2. For simplicity, as dual frequency laser source a continuous wave (CW) laser modulated in carrier suppression is employed. It is driven by a $20 \mathrm{GHz}$ oscillator, and a programmable clock divider provides the tunability of the modes' spacing.

On the reference path the frequency shift is obtained by means of an acousto-optic modulator (AOM) driven by a $40 \mathrm{MHz}$ RF source. On the other path the two laser modes are modulated with an RF synthesizer via a MZM biased in carrier suppression. The resulting optical signal is boosted up to $30 \mathrm{dBm}$ with an erbium doped fiber amplifier (EDFA), and it is sent to the target with a $3 \mathrm{~mm}$-aperture collimator. To avoid backreflections that could interfere with the phase estimation of the modulation tone, we avoid the circulator and collect the echo by another collimator with an aperture of $7 \mathrm{~mm}$, thus resorting to a bistatic implementation. The echo is coupled with the frequency shifted reference signal and then detected and amplified by an avalanche photodiode (APD) and its transimpedance amplifier (TIA). Finally a 100Msp/s 14-bit ADC digitized the photodiode's signal, as well as the modulation signal that will be used to determine the propagation phase delay. 


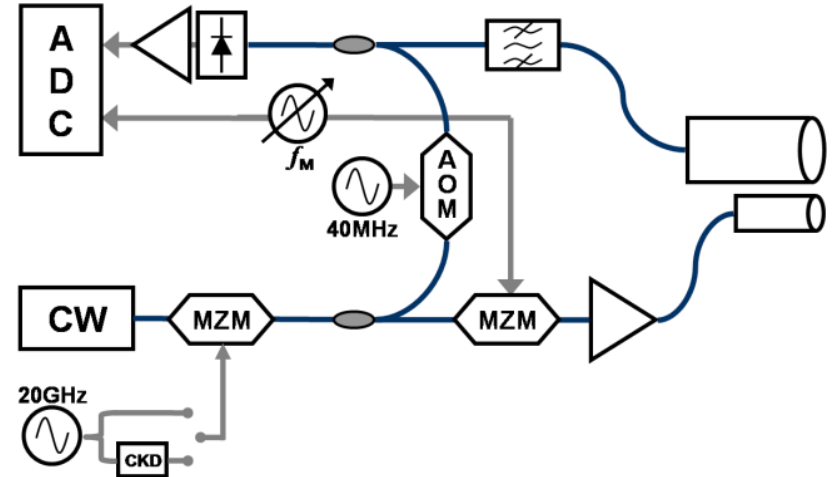

Fig. 2. Experimental setup. CW: Continuous Wave Laser, AOM: acousto optic modulator, MZM: Mach Zehnder modulator, ADC: analog to digital converter, CKD: Clock Divider.

The laser radar has been first tested targeting a static target at different positions to extract the range information and the relative precision of the measure. An example of the FFT spectrum of the acquired signal is depicted in Fig.3, employing $40 \mathrm{GHz}$ detuned modes (i.e. avoiding the clock divider) and a modulation frequency $f_{\mathrm{M}}=3 \mathrm{MHz}$. With respect to Fig.1-F, only two tones at $37 \mathrm{MHz}$ and $43 \mathrm{MHz}$ are present since the target is not moving, i.e. $\delta_{1}=\delta_{2}=0$. The FFT in Fig. 3 presents spurious tones due to the non ideality of the exploited test set up. In detail, a strong peak at $40 \mathrm{MHz}$ is caused by the non ideal carrier suppression of the MZM and the residual unshifted signal of the AOM. Moreover a weak tone at $6 \mathrm{MHz}$, generated by the direct detection of echo signal not amplified by the coherent detection, is also visible.

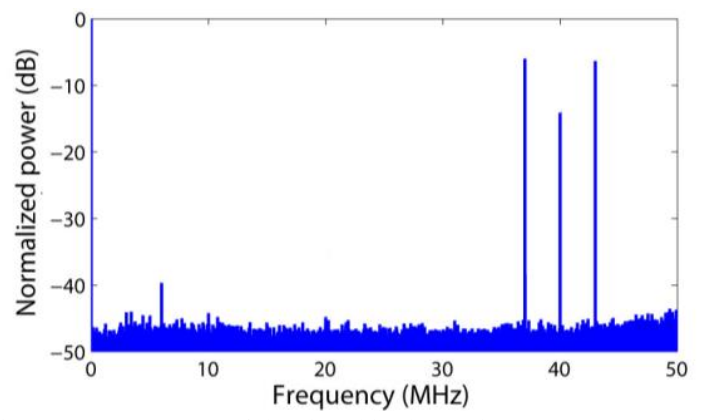

Fig. 3. FFT spectrum of the signal in the static target case, employing $\Delta f=40 \mathrm{GHz}$ and $f_{\mathrm{M}}=3 \mathrm{MHz}$.

Since these non idealities are known, they can be easily digitally erased before the squaring operation. In fact the range measurements confirm the effectiveness of the scheme. Fig.4 reports the measured ranges of the target with respect to its real positions, for different values of the modulation frequency. The coherent integration time was set to $500 \mathrm{~ms}$. As can be seen, the range precision increases with the modulation frequency. In fact, the range error $\zeta$ depends both on the signal to noise ratio (SNR) of the digitized signal as well as on $f_{\mathrm{M}}$ [26]:

$$
\zeta \cong \frac{\lambda / 2}{10^{\left(\frac{S N R}{20}\right)}}=\frac{c}{2 \cdot 10^{\left(\frac{S N R}{20}\right)} \cdot f_{M}}
$$

Nevertheless even with $f_{\mathrm{M}}=500 \mathrm{kHz}$, that gives an unambiguous range of $150 \mathrm{~m}$, the maximum occurred error is no greater than $1.4 \mathrm{~cm}$, while, with a modulation frequency of $6 \mathrm{MHz}(12.5 \mathrm{~m}$ of unambiguous range), this error is drastically reduced to $4 \mathrm{~mm}$. These results was obtained with a measured received power of about $-60 \mathrm{dBm}$.

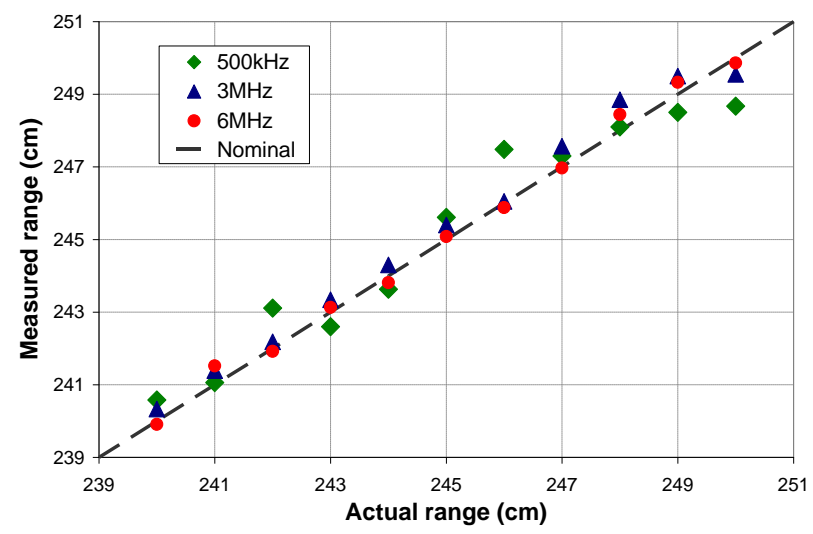

Fig. 4. Measured distances of the target with respect to its real positions, for different modulation frequencies.

The system has thus been tested varying the received power via a variable optical attenuator. The results are reported in Fig.5, that shows the standard deviation of $5010 \mathrm{~ms}-10 n g$ measures with a modulation frequency of $3 \mathrm{MHz}$, keeping the target stationary at about $1 \mathrm{~m}$ from the telescopes. A collected power of $-45 \mathrm{dBm}$ gives a standard deviation of only $350 \mu \mathrm{m}$. Reducing the power, the precision remains below $5 \mathrm{~cm}(0.2 \%$ of the unambiguous range) down to $-70 \mathrm{dBm}$, and could be still acceptable even at $-90 \mathrm{dBm}$. In fact, below this value the standard deviation rises up to about $70 \mathrm{~cm}$, i.e. $2.5 \%$ of the considered range.

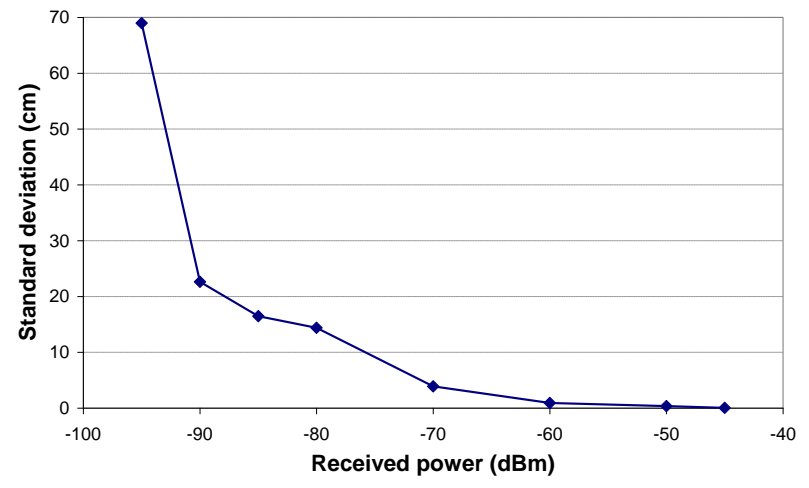

Fig. 5. Standard deviation of the measured range vs the optical power collected by the receiver telescope.

Beside the static characterization, the developed laser radar system has been tested in the detection of moving targets. To this extent, Fig.6-A reports the acquired spectrum in the case of a target under uniform rectilinear motion. As can be seen, with respect to Fig. $3 \delta_{1} \neq \delta_{2} \neq 0$ and the four tones detailed in (6) appear as two broadened spectral lines centered at $f_{\mathrm{IF}}+f_{\mathrm{M}}+\left(\delta_{2}+\delta_{1}\right) / 2$ and $f_{\mathrm{IF}}-f_{\mathrm{M}}+\left(\delta_{2}+\delta_{1}\right) / 2$ respectively. The inset in Fig.6-A shows these broadened spectral components. According to (5), since $\delta_{2}<\delta_{1}<0$, i.e. negative Doppler shift, 
the target is moving away with respect to the laser radar sensor. In fact, after squaring equation (6) the sign of the Doppler shift is lost, and it need to be evaluated here. To obtain a precise estimation of the velocity, a $100 \mathrm{~ms}$ portion of the temporal trace of the ADC output signal is squared, and the tone at $\left|\delta_{2}-\delta_{1}\right|$ is highlighted with a frequency resolution of $10 \mathrm{~Hz}$, as shown in Fig.6-B, so that its peak at $260 \mathrm{~Hz}$ can be appreciated. Since the employed emulated microwave frequency is $40 \mathrm{GHz}$, the measured Doppler shift corresponds to a velocity of $0.975 \mathrm{~m} / \mathrm{s}$. The position and velocity of the target over $1 \mathrm{~s}$, as measured by the developed system, are depicted in Fig.6-C. The range linearly increase according with the negative Doppler shift, and since in 1s it covers about $1 \mathrm{~m}$, a speed of $1 \mathrm{~m} / \mathrm{s}$ can be estimated. Considering the discrete $10 \mathrm{~Hz}$ resolution, i.e. an uncertainty of $0.0375 \mathrm{~m} / \mathrm{s}$ according to equation (12), this perfectly matches the measured Doppler.

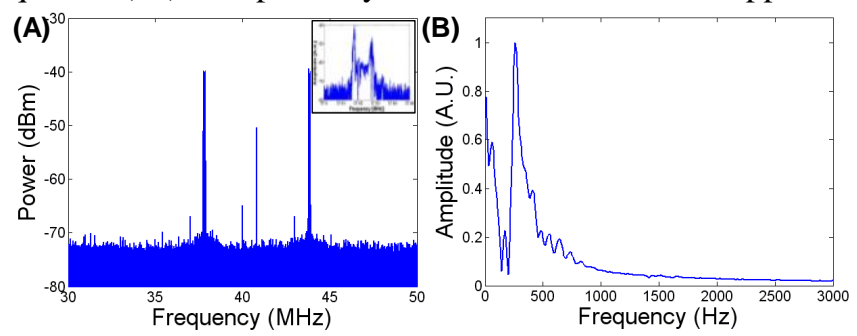

(C)

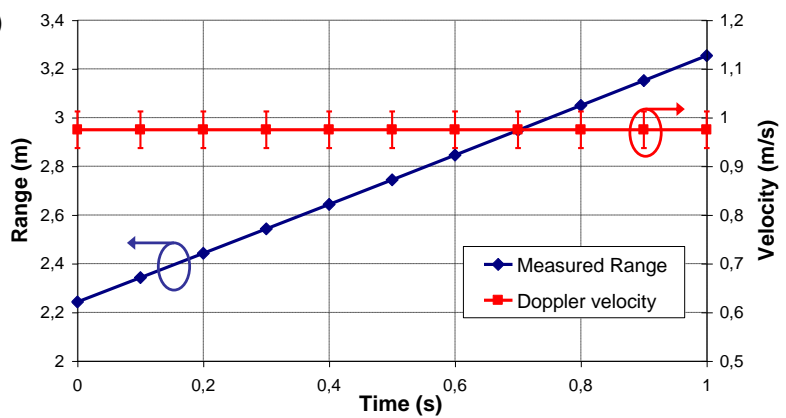

Fig. 6. A) FFT of the signal acquired by the ADC in the case of a moving target; B) FFT of the squared signal; C) Range and velocity as measured by the laser radar system.

Since the developed system has been demonstrated and characterized both with static and uniformly moving targets, to better prove its effectiveness further trials with randomly moving targets have been carried on. In these tests, reported in Fig.7, the integration time was reduced to $20 \mathrm{~ms}$ to avoid spreading in the Doppler spectrum, as well as in the range determination. This resulted in a reduced frequency resolution down to $50 \mathrm{~Hz}$, thus increasing the uncertainty of the measured velocity. Moreover to highlight the flexibility of the proposed architecture different parameters have been employed. For the measure in Fig.7-A the generated modes present a detuning of $20 \mathrm{GHz}$ (i.e. the RF oscillator is divided by a factor of 2), and a modulation frequency $f_{\mathrm{M}}=3 \mathrm{MHz}$ has been used. The second measure, reported in Fig.7-B, employed a mode spacing of $40 \mathrm{GHz}$ and a modulation frequency of $6 \mathrm{MHz}$. In both the experiments the target firstly moves away from the telescope, then comes back, and changes again its direction. The measured velocity always follows the range variations, in particular can be appreciated that whenever the direction of the motion changes, the velocity crosses the zero value. The finite frequency resolution affect the velocity measurement as visible in the resolution steps. Since the test range is about $16 \mathrm{~m}$ long and in the second measure the $f_{\mathrm{M}}=6 \mathrm{MHz}$ unambiguous distance results $12.5 \mathrm{~m}$, a portion of the trace is aliased at lower distance. Nevertheless this does not influence the measure of the velocity, which doesn't present discontinuities.

The proposed architecture thus demonstrated its capability to precisely measure both the range and the velocity of an arbitrary target, even with different $f_{\mathrm{M}}$ or $\Delta f$ configurations.
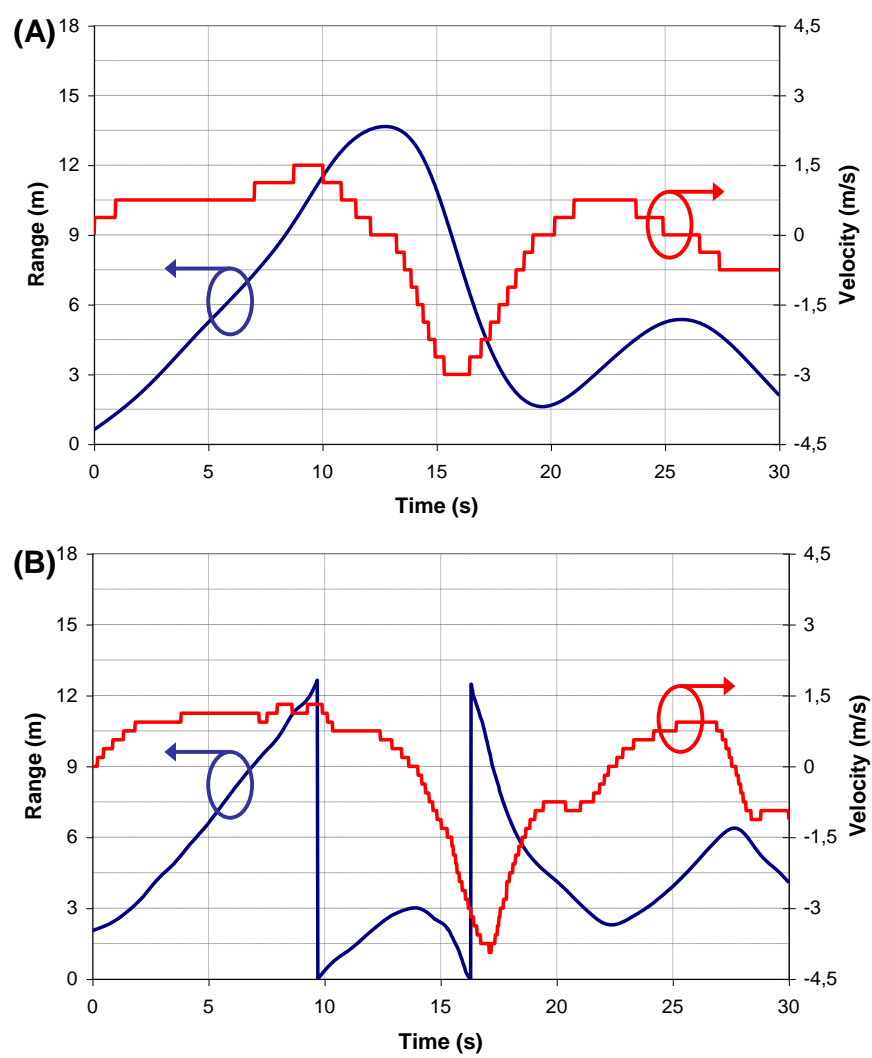

Fig. 7. A) Range and velocity measure of a randomly moving target, $\Delta f=20 \mathrm{GHz}$ and $f_{\mathrm{M}}=3 \mathrm{MHz} ; \mathrm{B}$ ) Range and velocity measure of a randomly moving target, $\Delta f=40 \mathrm{GHz}$ and $f_{\mathrm{M}}=6 \mathrm{MHz}$.

\section{DISCUSSION AND CONCLUSIONS}

In this paper, a novel architecture for a coherent interferometric dual frequency laser radar has been proposed, theoretically analyzed, and experimentally demonstrated. The scheme exploits the broadband tunability of a dual-wavelength optical source to precisely estimate the target velocity via differential Doppler measure, and the high stability of a low frequency RF source for the interferometric measure of the target range with extreme accuracy. This way, both the data are extracted from a single measure.

In particular, the DFL technique provides velocity information immune to the speckle noise induced by target's roughness and atmospheric turbulence, which causes spectral broadening and limits the resolution of the sensing system. Moreover the possibility to adjust the frequency spacing of the employed laser modes permits to adapt the system's resolution 
to the observed situation, e.g. reducing the emulated microwave frequency $\Delta f$ for vibrating targets or increasing it do detect very slow drifts. The interferometric approach for the range measure instead guarantees an extreme accuracy despite the narrow bandwidth, thus allowing the use of low sample rate digital electronics. Moreover the frequency modulation signal can be easily tuned, thus adapting the system range resolution and ambiguity to the scenario. In details, lower frequencies can be employed to roughly determine the target distance, and an higher frequency, with a reduced unambiguous range, can provide a precise measure.

Furthermore, the coherent detection avoids the noisy EDFA at the receiver stage, and increase the sensitivity with low optical echoes, allowing a size reduction of the focusing optics, thus reducing their weight and cost, and a lower transmitted power, thus increasing the safety and reducing the power consumption.

All these peculiarities has been experimentally validated, characterizing the system in different conditions. The spatial resolution reached millimeters accuracy and hundred meters unambiguous range. The velocity estimation matched the range variation with the only limit of the discrete FFT spectral resolution, providing a $10 \mathrm{~Hz}$ precision. Moreover the sensitivity measure demonstrated an error $<1 \%$ even with a collected received power of $-90 \mathrm{dBm}$.

Therefore, the validation of the proposed architecture demonstrates its suitability in applications where harsh environment requires high level of reconfigurability between precision and robustness.

\section{REFERENCES}

[1] P. F. McManamon, " Review of ladar: a historic, yet emerging, sensor technology with rich phenomenology ", Optical Engineering 51(6), 060901 (June 2012)

[2] M. Richards, J. A. Scheer, W. A. Holm., "Principle of Modern Radar: basic principle", SciTech Publishing, 2010.

[3] Richard D. Richmond, "Direct-detection LADAR Systems", SPIE Press, 2010

[4] S. Boudreau, S. Levasseur, C. Perilla, S. Roy, J. Genest, "Chemical detection with hyperspectral lidar using dual frequency combs" Optics Express, Vol. 21, No. 6, March 2013.

[5] P. Lutzmann et al., "Laser vibration sensing: overview and applications," Proc. SPIE 8186, 818602 (2011).

[6] M. A. Itzler et al., "Comparison of $3232 \times 128128$ and $3232 \times 3232$ Geiger-mode APD FPAs for single photon 3D LADAR imaging," Proc. SPIE 8033, 80330G (2011).

[7] M. Dierking et al., "Synthetic aperture LADAR for tactical imaging overview," Proc. 14th CLRC, Session 9 (2007).

[8] P. Gatt, S.W. Henderson, " Laser Radar Detection Statistics: A Comparison of Coherent and Direct Detection Receivers " Proc. on SPIE Vol. 4377 (2001).

[9] M.C. Amman, T.B.M. Lescure, R. Myllylä, M. Rioux, "Laser ranging: a critical review of usual techniques for distance measurement" Opt.Eng. 40(1), 10-19, January 2001.
[10] Wideband dual-frequency lidar-radar for simultaneous velocity and high-resolution range profile measurements, G. Pillet; L. Morvan, D. Dolfi, J.-P. Huignard Proc. SPIE 7323, Laser Radar Technology and Applications XIV, $73230 Z$ (May 2009).

[11] D. Kravitz, D. Grodensky, A. Zadok, N. Levanov, "Incoherent Compression of Complementary Code Pairs for Laser Ranging and Detection", 2013 IEEE International Conference on Microwaves, Communications, Antennas and Electronic Systems (COMCAS 2013), Tel Aviv, Israel, 21-23 October 2013.

[12] R. V. Chimenti, M. P. Dierking, P. E. Powers, J. W. Haus, "Sparse frequncy modulated LFM ladar signals", OPTICS EXPRESS 8302, Vol. 17, No. 10, May 2009

[13] S. Kameyama, T. Yanagisawa, T. Ando, T. Sakimura, H. Tanaka, Masashi Furuta, Y Hirano, "Development of wind sensing coherent Doppler LIDAR at Mitsubishi Electric Corporation from late 1990s to 2013", Proc. on. 17th Coherent Laser Radar Conference, 2013.

[14] A. Dolfi-Bouteyre, D. Goular, C. Planchat, S. Loaec , L. Thobois , J.P. Cariou , F. Barbaresco , P. Juge , F. Orlandi , Y. Ricci, M. Klein, " All-weather sensors (lidar + radar) for Wake-Vortex hazards mitigation on Airport", Proc. on. 17th Coherent Laser Radar Conference, 2013.

[15] L. Pichugina, R. M. Banta, W. A. Brewer, S. P. Sandberg, R. M. Hardesty, "Doppler Lidar-Based Wind-Profile Measurement System for Offshore Wind-Energy and Other Marine Boundary Layer Applications", Journal of applied meteorology and climatology, Vol. 51, Issue 2, 2012.

[16] G. Pappalardo, A. Comeron,, "ACTRIS: European aerosols, clouds, and trace gases research infraestructura network", Proc. on. 17th Coherent Laser Radar Conference, 2013.

[17] R. Diaz, S-C. Chan, J-M. Liu, "Lidar detection using a dual-frequency source", Optics Letters, 31, 3600-3602, (2006)

[18] M.-C. Haman, T. Bosch, M. Lescure, R. Myllylä, M. Rioux, "Laser ranging: a critical review of usual techniques for distance measurement", Optical Engineering, 40, 10-19, (2001)

[19] S. Gao, M. O'Sullivan, R. Hui, "Complex-optical-field lidar system for range and vector velocity measurement", Optics Express, 20, 2586725875, (2012).

[20] J M Vaughan, "Coherent Laser Spectroscopy and Doppler Lidar Sensing in the Atmosphere", Physica Scripta, T78, 73-81, (1998).

[21] C.-H. Cheng, J.-W. Lee, T.-W. Lin, F.-Y. Lin, "Speckle Noise Reduction of a Dual-Frequency Laser Doppler Velocimeter Based on an Optically Injected Semiconductor Laser", in Proc. SPIE. 8255, Physics and Simulation of Optoelectronic Devices XX, 82551P, (2012).

[22] M. U. Piracha, D. Nguyen, I. Ozdur, P. J Delfyett, "Simultaneous ranging and velocimetry of fast moving targets using oppositely chirped pulses from a mode-locked laser", Optics Express, 19, 11213-11219, (2011).

[23] F. Scotti, D. Onori, M. Scaffardi, E. Lazzeri, A. Bogoni, F. Laghezza; "Multi-Frequency Lidar/Radar Integrated System for Robust and Flexible Doppler Measurements", IEEE Photonics Technology Letters, DOI 10.1109/LPT.2015.2461458, 2015.

[24] M. Fujiwara, M. Teshima, J. Kani, "Optical Carrier Supply Module Using Flattened Optical Multicarrier Generation Based On Sinusoidal Amplitude and Phase Hybrid Modulation", IEEE Journal of Lightwave Technology, Vol 21, no 11, 2003

[25] T. B. Simpson and F. Doft, " Double-Locked Laser Diode for Microwave Photonics Applications", IEEE Photonic Technology Letters, Vol 11, no 11, 1999..

[26] W. C. Stone, M. Juberts, N. Dagalakis, J. Stone, J. Gorman, "Performance Analysis of Next-Generation LADAR for Manufacturing, Construction, and Mobility", NISTIR 7117, 2004. 